\title{
Summary of 14th Annual Saskatchewan Christmas Bird Count, 1955
}

\author{
By DR. STUART HOUSTON
}

The newly organized Saskatoon Natural History Society led the province with a one-day total of 23 species. The Regina group had the largest total for the Christmas season - 28 species. Greatest credit, however, should go to Joyce Gunn and Bill Anaka at Spirit Lake who saw 17 species in one day - all on foot through deep snow in a district where all roads were blocked.

All together 47 species were seen on the day of the count, and 6 additional during the Christmas season. Five species were recorded for the first time, making a total of 78 species that have been seen in Saskatchewan during the Christmas season, in 14 years. The open water at the Wascana sanctuary, Regina, produced the Pintail, Green-winged Teal, Canada Goose (captive) and the Whistling Swan (captive). Dr. R. M. Bremner at Saskatoon and Laurence Beckie at Bladworth both reported Pine Siskins. (Mrs. McDonald at Swan River reported a Great Gray Owl, but this is just outside Saskatchewan). The Richardson's Owl noted at Dilke by Margaret and J. B. Belcher was the second Saskatchewan Christmas record for this species in 14 years. The Hawk Owl at Spirit Lake was also of unusual interest.

Most frequently recorded species were, in order of occurrence, Magpie, Sharp-tailed Grouse English Sparrow, Black-capped Chickadee, Snow Bunting, Common Redpoll, Pine Grosbeak, Hairy Woodpecker, Downy Woodpecker and Hungarian Partridge. From this, it would seem that the Sharp-tails are well distributed this year.

The adverse weather conditions discouraged some of our regular Christmas counters this year, and we hope they will rejoin us next year.

ARDATH, December 22, by bus. 5 species, 51 indiv. Sharp-tailed Grouse, 8; Hungarian Partridge, 6; Canada Jay, 2; English Sparrow, 25; Snow Bunting, 10 - Ronald and Donald Hooper.
BIG RIVER, Dec. $30,1 / 2 \mathrm{mi}$. on foot. 10 species, 78 indiv. Pileated Woodpecker, 2; Hairy Woodpecker, 2; Downy Woodpecker, 7; Canada Jay, 5; Blue Jay, 12; Magpie, 4; Black-capped Chickadee, 25; Whitebreasted Nuthatch, 2; Common Redpoll, 16; Snow Bunting, 3; (Add: Raven, 2) - Mrs. Anne Olson.

BLADWORTH, Dec. 28, $5 \mathrm{mi}$. on foot, 2 hrs. 7 species, 317 indiv. Prairie Falcon, 1; Sharp-tailed Grouse 3; Hungarian Partridge, 28; Magpie, 4; English Sparrow, 2; Pine Grosbeak, 4; Snow Bunting, 275; (Add: Bohemian Waxwing, 11; Common Redpoll, 5; Pine Siskin, 2) - P. Laurence Beckie.

BROADVIEW, Dec. 25, $108 \mathrm{mi}$. by car in 3 hrs. 5 species, 17 indiv. Sharp-tailed Grouse, 5; Hungarian Partridge, 4; Snowy Owl, 1; Magpie, 1; Snow Bunting, 6; (Add: Great Horned Ow l, 1; Black-capped Chickadee, 1) - Charles and Audrey Thacker.

DILKE, Jan. 2, $30 \mathrm{mi}$. by car in $21 / 4$ hrs. 8 species, 191 indiv. Golden Eagle, 1; Sharp-tailed Grouse, 11; Hungarian Partridge, 40; Richardson's Owl, 1; Starling, 3; English Sparrow, 50; Common Redpoll, 55; Snow Bunting, 30; (Add: Snowy Owl, 1; Magpie, 1; Pine Grosbeak, 1) S. R., J. B., and Margaret Belcher.

ESTON, Dec. $21,6 \mathrm{mi}$. on foot in 3 hrs. 5 species, 167 indiv. Hungarian Partridge. 13; Snowy Owl, 1; Bohemian Waxwing, 60; English Sparrow, 80; Common Redpoll, 13; (Add: Snow Bunting, 6) — Ronald and Donald Hooper.

HAWARDEN, Dec. $31,1 \mathrm{mi}$. by team and on foot in 8 hrs. 5 species, 182 indiv. Goshawk, 1; Snowy Owl, 1; English Sparrow, 100; Common Redpoll, 10; Snow Bunting, 70; (Add: Golden Eagle, 1; Sharp-tailed Grouse, 3; Horned Owl, 1; Magpie, 1; Bohemian Waxwing, 18; Pine Grosbeak, 1) - Harold and Gerlsard Kvinge.

McLEAN, Dec. 30, $2 \mathrm{mi}$. on foot. 5 species, 19 indiv. Sharp-tailed Grouse, 6; Hairy Woodpecker, 1; Downy Woodpecker, 2; Black-capped 
Chickadee, 6; Bohemian Waxwing, 4. Mrs. Bray and Mrs. Newton.

MULLINGAR, Jan. 2, 2 mi. by team in $11 / 2$ hrs. 9 species. 66 indiv. Sharp-tailed Grouse, 1: Hairy Woodpecker, 2; Downy Woodpecker, 1; Canada Jay, 1; Magpie, 2; Blackcapped Chickadee, 6; English Sparrow, 17; Common Redpoll, 15; Snow Bunting, '21; (Add: Ruffed Grouse, 2; Pine Grosbeak, 12) - Ralph T. Cowell.

NAICAM, Dec. $29,5 \mathrm{mi}$. on foot in 7 hours. 5 species, 23 indiv. Hungarian Partridge, 1; Magpie, 5; Blackcapped Chickadee, 4; English Sparrow, 7; Pine Grosbeak, 6. (Add: Downy Woodpecker, 1; Common Redpoll, 5; Snow Bunting, 75) W. Yanchinski.

PUNNICHY, Dec. 30,6 mi. by team. 6 species, 41 indiv. Ruffed Grouse, 1; Horned Owl. 2; Hairy Woodpecker, 2; Downy Woodpecker, 1; Black-capped Chickadee, 7: English Sparrow, 28: (Add: Sharptailed Grouse, 6; Magpie, 2; Crow, 1) - Carl and Madeline Runyan.

REGINA, Dec, 27, 8 hrs. 21" species, 530 indiv. Pied-billed Grebe, 2; Whistling Swan. 2: Canada Goose, 20; Mallard, 175; Pintail, 3; Pigeon Hawk, 1; Sharp-tailed Grouse, 33; Hungarian Partridge, 5; Coot, 1; Horned Owl, 1; Downy Woodpecker, 3; Magpie, 7; Black-capped Chickadee. 2 ; Brown-capped Chickadee, 3; Red-breasted Nuthatch, 1; Bohemian Waxwing, 160; Northern Shrike, 1; English Sparrow, 50; Pine Grosbeak, 7; Common Redpoll, 40: Whitewinged Crossbill, 13; (Add: Greenwinged Teal, 1; American Goldeneye, 5; American Merganser, 1; Snowy Owl, 1; Hairy Woodpeaker, 1; White-breasted Nuthatch, 1; Hoary Redpoll, 300) - F. Brazier, E. Cruickshank, D. Gilroy, F. Lahrman, G. Ledingham, R. Nero, and E. Fox (compiler).

SASKATOON, Dec. 26, $112 \mathrm{mi}$. by car in $16 \mathrm{hrs}$; $24 \mathrm{mi}$. by foot in 21 hrs. 23 species, 3562 indiv. Lesser Scaup, 2; American Golden-eye, 2; Goshawk, 2; Sharp-tailed Grouse, 10; Hungarian Partridge, 109; Ringnecked Pheasant, 1; Horned Owl, 4; Snowy Owl, 2; Hairy Woodpecker, 2; Downy Woodpecker, 2; Canada Jay, 4; Magpie, 51; Crow, 2; Blackcapped Chickadee, 28; Bohemian Waxwing, 116; Cedar Waxwing, 40;
Northern Shrike, 2; Starling, 10; English Sparrow, 1187; Pine Grosbeak, 118; Common Redpoll, 1072; Pine Siskin, 4; Snow Bunting, 792. J. Agar, Dr. R. M. Bremner, Jean Davidson, F. J. H. Fredeen, Mrs. J. Gerrard, Jonathan Gerrard, J. B. Gollop, J. D. Hogg, Dr. C. J. Houston, Dr. and Mrs. Stuart Houston (compilers), John Hunter, Bob Mills, Dr. Lucy Murray, M. W. Nickel, Bob Pravda, Ed. Reed, J. F. Roy, J. Shadidk, Keith Thue, John Webster, Reuben Willems, F. J. Wilson, Dr. Keith Yonge (Saskatoon Natural History Society).

SHEHO, Dec. $31,3 \mathrm{mi}$. by car and $6 \mathrm{hrs}$. on foot. 12 species, 172 indiv. Ruffed Grouse, 2; Sharp-tailed Grouse, 16; Horned Owl, 1; Hairy Woodpecker, 1; Downy Woodpecker, 2; Canada Jay, 1; Magpie, 5; Blackcapped Chickadee, 5; Evening Crosbeak, 7; Pine Grosbeak, 16; Common Redpoll, 16; Snow Bunting, 100. William Niven.

SKULL CREEK, Dec. 26, $4 \mathrm{mi}$. by car, $4 \mathrm{mi}$. on foot, in $3 \mathrm{hrs}, 12$ species, 399 indiv. Mallard, 5; Golden Eagle, 1; Hungarian Partridge, 3; Ring-necked Pheasant, 7; Blue Jay, 3; Magpie, 21; Black-capped Chickadee, 14; Robin, 1; Bohemian Waxwing, 147; English Sparrow, 110; Common Redpoll, 84; Tree Sparrow, 3; (Add: Goshawk, 1; American Rough-legged Hawk, 1; Sharp-tailed Grouse, 60; Short-eared Owl, 1; Horned Lark, 39; Northern Shrike, 1; Snow Bunting) - S. A. Mann, Helen Mann, Peter Swain, Verna Swain, George Swain.

SOMME, Dec. $24,6 \mathrm{hrs}$, on foot. 9 species, 103 indiv. Horned Owl, 1; Hairy Woodpecker, 1; Magpie, 1; Raven, 2; Black-capped Chickadee, 1; English Sparrow, 60; Pine Grosbeak, 6; Hoary Redpoll, 1; Snow Bunting, 30: (Add: Ruffed Grouse, 3; Sharp-tailed Grouse, 1; Hungarian Partridge, 7; Blue Jay, 6; Common Redpoll, 4). - Ronald and Donald Hooper.

SPIRIT LAKE, Jan. 1, 61/2 mi. on foot in $7 \mathrm{hrs} .17$ species, 163 indiv. Ruffed Grouse, 3; Sharp-tailed Grouse, 2; Horned Owl, 2; Hawk Owl, 1; Hairy Woodpecker, 6; Downy Woodpecker, 8; Canada Jay, 4; Blue Jay, 3; Magpie, 2; Black-capped Chickadee, 46; White-breasted Nuthatch, 3; English Sparrow, 25; 
Evening Grosbeak, 6; Pine Grosbeak, 4; Common Redpoll, 5; White-winged Crossbill, 3; Snow Bunting, 40; (Add: Snowy Owl, 1) — Joyce Gunn, Bill Anaka.

SWAN RIVER, Dec. 26, $20 \mathrm{mi}$. by car. 16 species, $\mathbf{5 0 8}$ indiv. Hairy Woodpecker, 2; Downy Woodpecker, 2; Canada Jay, 3; Blue Jay, 6; Magpie, 2; Black-capped Chickadee, 16; Brown-capped Chickadee, 1; Whitebreasted Nuthatch, 1; English Sparrow, 22; Evening Grosbeak, 36; Pine Grosbeak, 2; Hoary Redpoll, 3; Common Redpoll. 16; Red Crossbill, 4; White-wirged Crossbill, 17; Snow Bunting, 375. (Add: Sharp-tailed Grouse, 1; Snowy Owl, 1; Great Gray Owl, 1; Pileated Woodpecker, 1; Raven, 5) - Mr. and Mrs. J. H. McDonald.

WYNYARD. Jan. 3.8 species, 60 indiv. Hairy Woodpecker, 2; Downy Woodpecker, 2; Canada Jay, 1; Blue Jay. 1; Magpie, 3; Black-capped Chickadee, 10: English Sparrow, 30; Pine Grosbeak, 11. (Add: Evening Grosbeak, 2) - D. Bardal.

YORKTON, Dec. 26, $66 \mathrm{mi}$. by car in $4 \frac{1}{2} \mathrm{hrs}$., $10 \mathrm{mi}$. on foot in $4 \mathrm{hrs}$. 11 species, 415 indiv. Sharp-tailed Grouse, 22; Snowy Owl. 1; Hairy Woodpecker, 2; Downy Woodpecker, 1: Blue Jay, 1; Black-capped Chickadee, 8; Bohemian Waxwing, 7: Starling. 6; English Sparrow, 143; Pine Grosbeak, 7; Snow Bunting, 217. - Mr. and Mrs. Art Gellert;

Brother Clarence: Paul Welgan; Lionel Coleman; Cliff, Doug and Karen Shaw.

\section{The American Egret}

(Casmerodius albus egretta) NESTING IN MANITOBA

By RALPH D. BIRD, Brandon, Man.

Mr. E. L. Fox's article on the American Egret in the Oct.-Nov.Dec. 1955 number of The Blue Jay has stimulated me to round out records on its occurrence and breeding by a short note on this bird in Manitoba. In 1954 and 1955 the American Egret appeared in all three prairie provinces. Oeming and Riggall (Canadian Field-Nat. 69(2):6768 ) record the first occurrence of the bird in Alberta at Cowley and Edmonton in 1954. Fox records it nesting in Saskatchewan in 1955 ,

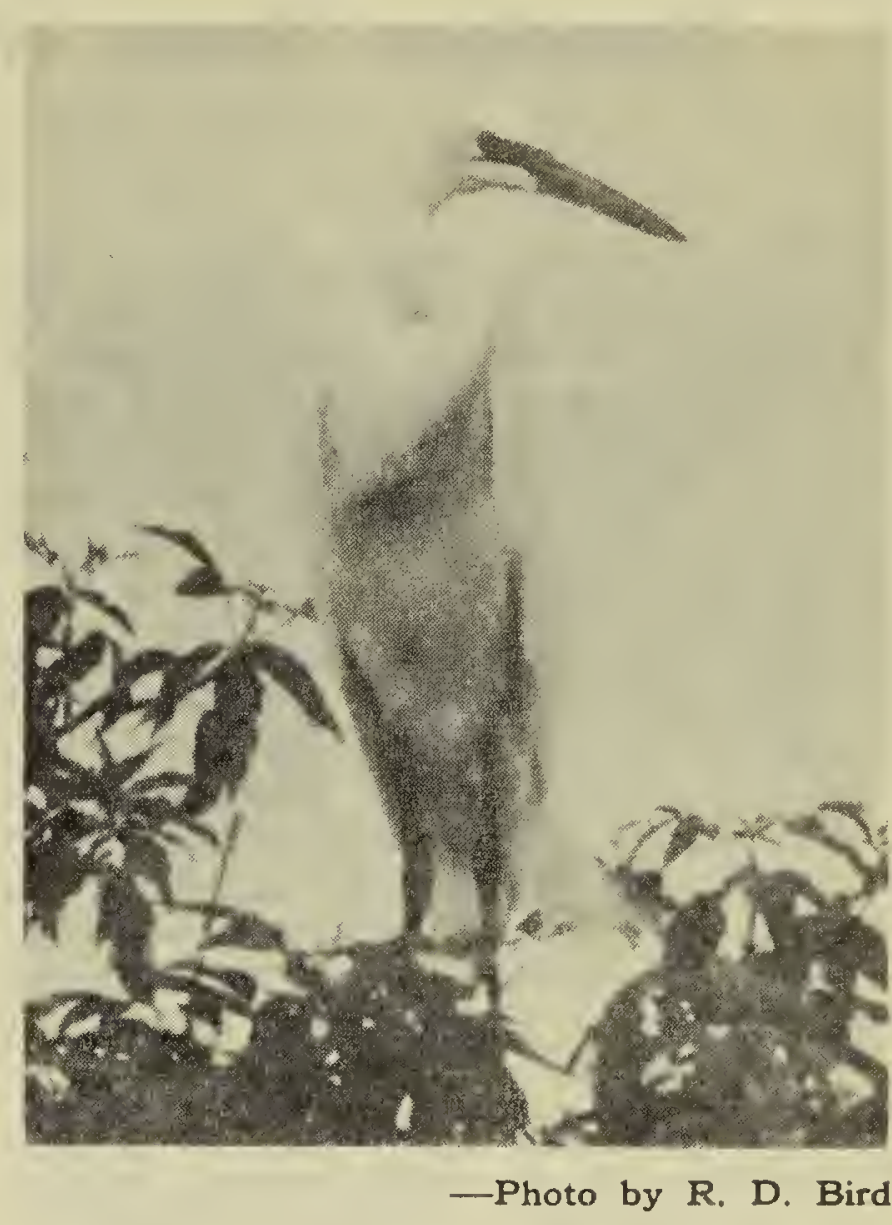

and I have just submitted an article to The Canadian Field-Naturalist recording it nesting in Manitoba in the same year. Previous to this there have been only four sight records of the bird in Manitoba.

On May 10, 1955, I observed a single egret feeding on flooded flats of the Souris River near Napinka, Man. On June $5 \mathrm{I}$ saw a single bird among a colony of Great Blue and Black-crowned Night Herons near Pipestone, and on returning on June 19 I found it had a nest and young. One bird was wary but the other, presumably the female, was quite tame and permitted me to obtain a number of photographs, one of which is included. On August 12 Mrs. James Stewart of Napinka saw an adult and three young close to the location where I had seen the single bird in May and only fifteen airline miles from the nesting site.

The number of recent egret records gives evidence of a possible northward extension of breeding range.

Another recent occurrence of a southern bird is a female Redbellied Woodpecker, which I observed at the feeding station of Mrs. Cliff Chapman, ten miles southeast of Brandon, on New Year's Day 1956. There are only a few previous records of this bird in Manitoba. 